\title{
Research
}

\section{Resilience of primary healthcare professionals working in challenging environments:}

\author{
a focus group study
}

\begin{abstract}
Background

The modern primary healthcare workforce needs to be resilient. Early research framed professional resilience as avoiding 'burnout'; however, more recent literature has introduced the concept of positive adaptation to professional challenges, which results in individuals thriving in their role.

\section{Aim}

To explore what primary health professionals working in challenging environments consider to be characteristics of resilience and what promotes or challenges professional resilience.
\end{abstract}

\section{Design and setting}

A qualitative focus group in north east Scotland.

\section{Method}

Five focus groups were held with 20 health professionals (six GPs, nine nurses, four pharmacists, and a practice manager) based in rural or deprived city areas in the north east of Scotland. Inductive thematic analysis identified emerging themes.

\section{Results}

Personal resilience characteristics identified were optimism, flexibility and adaptability, initiative, tolerance, organisational skills, being a team worker, keeping within professional boundaries, assertiveness, humour, and a sense of self-worth. Workplace challenges were workload, information overload, time pressures, poor communication, challenging patients, and environmental factors (rural location). Promoters of professional resilience were strong management support, teamwork, workplace buffers, and social factors such as friends, family, and leisure activities.

\section{Conclusion}

A model of health professional resilience is proposed that concurs with existing literature but adds the concept of personal traits being synergistic with workplace features and social networks. These facilitate adaptability and enable individual health professionals to cope with adversity that is inevitably part of the everyday experience of those working in challenging healthcare environments.

\section{Keywords}

health professionals; multiple deprivation; primary care; qualitative research; resilience, psychological.; rurality.

\section{INTRODUCTION}

The role of modern primary healthcare professionals is a challenging one and resilience is increasingly recognised as a key requisite. ${ }^{1}$ Primary or communitybased health care has particular cultural and environmental factors that may affect the resilience of healthcare professionals. Resilience has been described as a dynamic process encompassing positive adaptation within the context of significant adversity.? However, for an individual to be able to demonstrate resilient qualities, they must first have encountered adversity at work. ${ }^{2}$ Definitions of resilience in the context of being a primary care professional include the ability to overcome or bounce back' from the negative effects of stresses and challenges, and to remain positive in the face of adversity. ${ }^{3}$

Personal resilience has been much studied in the psychology literature. Resilience in health professionals has been investigated, albeit to a lesser extent, because health care can be particularly stressful as a result of frequent challenging situations. A literature review of nursing resilience and coping with workplace adversity concluded that resilience can be developed or strengthened, and that resilience development could be incorporated into nursing training. Resilience in a range of physicians has been studied in Germany ${ }^{4}$ and Australia. ${ }^{5,6}$ These

C Matheson, BSc, MSc, PhD, senior research fellow (honorary); HD Robertson, BSc Health Sci, research assistant; AM Elliott BSc, PhD, senior research fellow; L Iversen, $\mathrm{BSc}$ (Hons), MSc, PhD, DLSHTM, research fellow; P Murchie, MSc, PhD, FRCGP, clinical senior lecturer and GP; Academic Primary Care, Institute of Applied Health Sciences, University of Aberdeen, Aberdeen.

\section{Address for correspondence}

Catriona Matheson, Academic Primary Care, Institute of Applied Health Sciences, University of studies identified features of 'resilient' practitioners such as positive attitudes towards the patient population and realistic role expectations, as well as strategies to minimise stress such as leisure activities.

A recent literature review on resilience in primary healthcare professionals found resilience combined discrete personal traits and experience, leading to positive adaptation. ${ }^{7}$ Much of the previous evidence base framed health professionals' resilience in relation to avoiding 'burnout'. ${ }^{-10}$ Health professionals' resilience is clearly of global importance, although every country will have its own challenges depending on the structure of its healthcare system. Two areas previously identified as particularly challenging for primary care are working in areas of multiple deprivation, ${ }^{11}$ and working in remote and rural areas. ${ }^{12}$ Poor resilience leads to high staff turnover, which in turn impacts services for deprived and rural populations.

However, some health professionals thrive in challenging environments. It would therefore be useful to study such resilient practitioners to determine what it is about them or their environment that enables them to thrive. As Bowden and colleagues noted, ${ }^{13}$ there is an increasing need for health professionals to develop effective strategies to foster resilience to counteract challenges, and to enable them to continue to thrive in their roles. This

Aberdeen, Polwarth Building, Foresterhill, Aberdeen AB25 2ZD, UK.

E-mail: catriona.mathesondabdn.ac.uk

Submitted: 15 December 2015; Editor's response:

8 January 2016; final acceptance: 25 February 2016. (CBritish Journal of General Practice

This is the full-length article (published online 10 May 2016) of an abridged version published in print. Cite this version as: Br J Gen Pract 2016; DOI: 10.3399/bjgp16X685285 


\section{How this fits in}

Research to date has explored the resilience of health professionals. This study aimed to further our understanding by considering the individual in the context of their working environment. Individual characteristics were found to work synergistically with features of the workplace and social networks. A model of professional resilience in primary care in presented.

leads to consideration of whether health professionals' resilience can be developed to improve workforce sustainability.

This study aimed to explore what health professionals working in challenging environments such as rural areas or areas of multiple deprivation considered to be characteristics of resilience, and what promoted or challenged their professional resilience. From this, a model of health professional resilience is proposed.

\section{METHOD}

A qualitative focus group methodology was used because it is an efficient way to collect data and also because the generation of ideas that others can reflect on, which some participants may not have explicitly thought about previously, was considered ideal for this topic. The study took place in the north east of Scotland, which incorporates a large rural area and a city with areas of deprivation. Purposive sampling was used to include a range of staff with patient contact, based in the community, who care for patients who do not come under secondary care (GPs, practice nurses, district nurses, and pharmacists), and who routinely work in areas of deprivation or rurality. Potential participants were identified using the Scottish Index of Multiple Deprivation ${ }^{14}$ and Scottish Government Urban/Rural Classification $^{15}$ for their practice location. Travel cost reimbursements were offered

\section{Table 1. Characteristics of focus group participants}

\begin{tabular}{lccc} 
Group & Number of participants & Profession & Location \\
\hline Group 1 & 5 (2 male/3 female) & 2 GPs, 3 health visitors & Urban \\
Group 2 & 3 (all female) & 3 nurse practitioners & Urban \\
Group 3 & 6 (1 male/5 female) & 3 GPs, 1 district nurse, 1 practice & Rural \\
& & manager, 1 practice nurse & \\
Group 4 & $3(2$ male/1 female) & $1 \mathrm{GP}, 1$ district nurse, 1 pharmacist & Rural \\
Group 5 & $3(1$ male/2 female) & 3 pharmacists & Urban \\
\hline
\end{tabular}

but no payment or incentive, and lunch/ refreshments were provided. Initially, 15 practices ( 10 classified as deprived ${ }^{14}$ and five in areas classified as remote/rurall ${ }^{15}$ and 14 community pharmacies from deprived areas were contacted, and two pharmacists in rural locations invited to participate.

In line with an inductive qualitative approach, the topic guide lused by the facilitator) was deliberately minimalist and covered:

- What did participants consider to be resilience characteristics of health professionals?

- What did participants believe enabled or threatened professional resilience?

- Could resilience be developed or learnt?

Groups were facilitated by a senior researcher accompanied by a research assistant. Each participant was asked for written consent before taking part in the focus group. Discussions were digitally recorded and transcribed. Three researchers independently coded the first two transcripts. A thematic framework was agreed by consensus then applied across all focus group data.

\section{RESULTS}

Twenty participants attended five focus groups (characteristics are outlined in Table 1). The first four focus groups were held at general practice locations, one of which was attended by two nurses from a different practice, and the final session was held on university premises. Each focus group (FG) lasted for 45-60 minutes.

In line with the topic guide, four themes were discussed: features of a resilient health professional; challenges to resilience; promoters of resilience; and developing resilience. Within each of these high-level themes there were emergent subthemes, which are presented in detail below using illustrative verbatim quotes.

Features of a resilient health professional A number of characteristics of resilient health professionals were suggested, including optimism, flexibility and adaptability, initiative, tolerance, organisational skills, being a team worker, keeping within professional boundaries, confidence/assertiveness, humour, and a sense of self-worth.

Optimism was noted as a key feature of a resilient health professional:

'But it's also looking at things in a kind of positive light, it's not being, eh, drummed 
down, it's looking at it as "Well I can solve this", it's looking at the cup half full l'd say. It's "I can solve this".' (FG5-17)

Resilient individuals understood that the ability to be flexible and adaptable are essential in the health professional's role. This was especially raised in both focus groups in rural locations. These health professionals may have to deal with unscheduled care for drop-in patients when there is no access to the patients medical histories. Therefore rural health professionals must be prepared to deal with everything that turns up:

'Here you've to really be a Jack of all trades. (FG3-14)

'You just have to be adaptable, you can't work within the confines because then the job just doesn't work.' (FG4-16)

Adaptability was also evident in how participants described managing difficult situations such as a mistake being made or a confrontational encounter. This was raised by a pharmacist participant:

It would be very easy to carry these things [that is, a difficult situation] home, or carry it into the next prescription even, you know ... You have to be able to sort of deal with it, shut it off, compartmentalise it, move onto the next thing.' (FG5-17)

This ability to focus and deal with a problem, then move on, or 'bounce back', was important in the face of adversity, as was also noted by a GP participant:

I would relate it to the stress involved with the job, I believe that we can bounce back in our jobs, when I've been stretched, that's what as I see resilience in this job.' (FG1-04)

Using initiative was considered important to resilience. This was particularly noted by rural practitioners:

'I think, for being in a rural practice, you've got to, you do have to use your own initiative a lot.'(FG3-11)

Participants felt resilient individuals would anticipate certain situations and how they would react and deal with them:

'Rather than myselfbecoming overwhelmed and stressed at the idea of, I'm just gonna do this then and sort of anticipate this and you know...' (FG1-03)
However, using initiative in difficult situations was seen to require confidence. This was described as a type of 'arrogance' by one pharmacist when reflecting on himself:

I think there's an element too, I think of, arrogance and confidence, with resilience that you come across the issue and you're arrogant enough and confident enough that you can come up with a solution to solve it and to cope with whatever issue it is that comes through the door ... Arrogant in a good way / think.' (FG5-17)

A tolerant attitude was also noted, which is important in a team environment and when dealing with patients:

I think definitely tolerance as well. I think I'm definitely more tolerant the older I've become.' (FG2-08)

Good organisational skills were considered important to maintain a high quality of patient care. This included the ability to plan and prioritise workloads to achieve balance at work and manage time effectively:

'Yes, resilience can be time management as well, deciding ... prioritising your workload in a way that you maybe feel satisfied with. (FG1-01)

This concept was further expanded on by a participant explaining that efficient organisation in the workplace was important:

'Being organised also greatly helps, like it can kind of give us the strength to kind of reduce our workload and kind of be more organised so that we don't kind of repeat things.' (FG1-04)

Fostering good working relationships, with good communication pathways was considered important:

A robust way of working, a good way of working and good communication through that network.' (FG1-01)

Definitely, to share and communicate with others helps you through.' (FG1-05)

This raised the concept of teamwork, which was also evident in another focus group exchange:

'But it is a case of going into [the workplace] and if support staff there are good and they're resilient to a degree, you can be 
resilient. If they're not resilient or not on board or not on side with you, then it's a real struggle. '(FG5-17)

'You can't be resilient on your own, can you?' (FG5-17)

Having a clear understanding of professional boundaries can mitigate the volume and intensity of the workload, as identified by participants:

'Knowing your professional identity as well I think, isn't it, you know. You know what your job is. '(FG1-05)

Thus having the confidence to be assertive was raised as a feature of resilience, in relation to understanding one's role and professional identity, and when dealing with patients and other health professionals:

Knowing what your role is and sticking to that, I suppose being assertive with other disciplines.' (FG1-03)

There was also a caution that confidence and assertiveness did not turn into aggression and the idea of personal traits as features of resilience and the use of humour also emerged at this point:

'But you would need to have the right manner to do that. You need to have a lot of humour, you need a lot of kind of ... personal skills to be able to, so that the assertiveness doesn't become aggressive. (FG1-02)

Having the confidence to accept professional limitations, being able to say 'no', and being comfortable with that reality, came with experience:

'You don't stop caring, you become aware of your limitations and you become aware, you're not Superwoman and you're not Superdoctor that can do 24 hours a day, 365 , and there has to be, you have to say, "Well, I've done all I can do and that's it". And there's a big confidence thing about learning that.'(FG3-12)

Being able to appreciate and express humour at work was raised in several groups:

'You try and work together to find the best way forward. You can laugh about it though. (FG4-16)

'I think you have to have a sense of humour. (FG5-18)
Finally, the value that health professionals place on themselves and belief in their knowledge and experience helps them move beyond defining their self-worth externally, thereby building and strengthening their resilience:

You have to value yourself sort of as well, and the working, the contribution you make as well to the service ... probably internal and external, yeah, but I think you have to, em, feel it within yourself also you know. (FG1-05)

\section{Challenges to resilience}

Themes that emerged when asked about challenges or threats to resilience were around workplace challenges and included information overload, time pressures, and environmental features, particularly associated with rural working.

The issue of workload including information overload and volumes of paperwork while managing the practicalities of patient care was raised in all groups:

$\therefore$ and you switch on that silly computer and there's a thousand e-mails that seem, and possibly are, totally irrelevant in the big picture of things ... you know when you come back with all that in your head [clinical work] you just want a bit of TLC don't you? You want a little bit of time just to reflect a little bit of the time, and space just to like, deal with, them, so it's keeping all that rest of that at bay.' (FG1-02)

\section{And in another group:}

'There's so much paperwork in the health service across all disciplines.' (FG4-16)

Time pressures had a big impact on professional resilience, including limited patient appointment times and the view that workers need sufficient breaks during the day to maintain concentration:

I suppose patients coming in with multiple problems can stretch your resilience because you think you know, you've got your time, your appointment time to deal with. '(FG3-13)

There were rural-specific challenges mentioned in both rural focus groups. Rural doctors felt that patient types and demands were different compared with urban groups:

'The patient type, the epidemiology of the patient type in general in [rural location] is 
different to in town. People from farming backgrounds don't want to trouble the doctor ... and then it turns out that they've got something quite serious that's presenting quite late. Because you haven't seen them very often there's a lot more work to be done. (FG3-13)

The lack of resources, particularly access to other services, in remote areas and its impact on patient care was highlighted as a challenge:

'There's not as many resources for us to go to rurally. If we want to, we can't do exercise referrals or, you know, it's not so easy to access smoking cessation and things like that whereas that was all quite locally based in town. There's no, em, sort of, mental health services in [place].' (FG3-13)

\section{Promoters of resilience}

Suggested enablers of resilience were organisational structures, which included subthemes of strong management support, a team culture, and capacity for 'buffering', and work-life balance, which includes supportive home life and recreational activities.

Good organisational structures including contingency planning for staff shortages in the workplace were identified as important enablers of resilience:

'Because we don't have a big team, we cannot leave things, we need to prioritise our work, em, a lot more. We've always got contingency planning.' (FG3-11)

One of the important keys to organisational resilience was seen as strong management support:

I think the key to our, to the practice though is, em, we had a previous boss, Dr F, and N's the same, very supportive. I think if we didn't have that the place would fall down because there are so few of us and we definitely need support. So that's key to a small practice.' (FG3-11)

A team approach was described as invaluable for maintaining resilience. Having the knowledge that they can discuss issues and problems with colleagues helped build coping mechanisms:

How your day is structured, how everybody works together, and that I think creates a much more resilient work force in terms of the practice but also individually, it reinforces your own resilience.' (FG3-13)
The team approach, working closely with colleagues as well as the wider team, was perceived as helping absorb daily stresses while allowing the continuation of highquality patient care:

Having buffer mechanisms to, kind of, absorb the shocks that kind of, we get to see on a day-to-day basis. Em, I think for example, if one of our partners is off sick, that puts a lot of stress on the way we work, eh, but our practice is kind of built in such a way that we kind of have ways of dealing with these unseen circumstances. '(FG1-04)

Supportive colleagues also alleviated the pressures of lone working for district nurses and those in rural areas:

And then we try and meet again, em, in the afternoon, whoever's around. So we always come back to the base. So we all try and touch, touch base ... it's a safe environment ... you can express anything and it stays within the walls.' (FG3-14)

Resilience was further enabled through a good balance between work and the rest of life. This included the need to have a clear boundary between the two:

'I suppose that you don't let, I suppose part of it is maintaining a work-life balance and you're able to, em, not let work all consume you I suppose. To maintain resilience and keep a boundary of sort of, of where your life is and where your work starts, does that make sense?'(FG3-13)

Participants discussed the link between resilient health professionals and supportive personal and social activities:

'Personally, I make sure I get enough sleep, I have a decent sort of work-life balance, plan things for my time off, sort of, holidays, gettin' away from what I do on a day-today basis, mates, meeting up with friends, family. Eating relatively sort of healthy, exercise, hobbies.' (FG1-03)

There were other further discussions around the support of friends and family with regard to aiding resilience and coping with work adversity:

'If you've got good support at home or socially, then you can always cope with things better.' (FG3-13)

\section{Developing resilience}

All participants agreed that resilience 
could be developed even if it was not a natural trait. When asked how resilience could be developed, emerging themes were experience, learning from others, and formal training.

General exposure to and experience of different and challenging situations was a strong theme across groups, as this exchange illustrated:

And exposure to different situations.' (FG206)

Yes, difficult situations, good situations. But I think it's the more challenging situations that builds up the resilience. '(FG2-08)

\section{'I would agree.'(FG2-07)}

This gave a sense of naturally developing resilience with increasing maturity, experience of adversity, and familiarity with workplace expectations. This was expanded on in another group that linked it to confidence:

'But there's no doubt, through your career you become more resilient, you know. As a newly-qualified staff nurse or a newlyqualified whatever, you know you're much more vulnerable to pressures and what people say and how people react and you're much more constrained by, you know "I haven't managed to do this today", and you feel terrible about it and all the rest of it. But as you get older ... you are more mature, you do become more ... yeah, more comfortable with yourself.' (FG3-14)

Resilience was also perceived as something that could fluctuate throughout a health professional's career, along with changing perspectives:

For me, looking back, my resilience has definitely fluctuated at different times ... I was off [career break] and you come back and you've lost confidence and things have changed a bit. Or you've moved jobs and getting to grips with new things, em, to build up your resilience again.' (FG3-13)

The concept of willingness to learn from others' experiences was raised. Recognising resilience in more experienced colleagues was noted as a way to develop resilience:

And I think sometimes looking at colleagues, looking at, at how they ... work and operate, em, sometimes you can learn from that. So rather than get overwhelmed, if your workload changes and you suddenly have to drop everything and do something that you weren't expecting to do. To sort of see a bigger picture and maybe sort of learn from observing sort of how colleagues, how other people manage it.'(FG1-03)

The importance of ongoing professional training was recognised as nurturing health professionals' resilience, in terms of building and learning new skills, and maintaining contact with other colleagues:

And training sort of helps with that as well you know ... even refresher courses and things like that. It gives you extra confidence. (FG3-13)

Although it was agreed that some individuals had more intrinsic resilience, it was suggested that health professionals can be trained to gain skills to manage difficult situations. Training mentioned included mindfulness, briefinterventions, motivational interviewing, and communication skills as potential aids to increasing resilience:

I think you have to have the skills, so I guess there's elements of, em, training and knowledge come in there as well.' (FG5-18)

'Yes, there's an element of that, if you're not a resilient person, you can be taught skills that will stop you getting into a position where you then have to be a resilient person ... like brief intervention skills, motivational interviewing, and all those kind of communication skills. (FG5-17)

\section{DISCUSSION}

\section{Summary}

Participants in this study discussed resilience in relation to four themes: features of a resilient health professional; challenges to resilience; promoters of resilience; and developing resilience. Key ingredients of features of resilience included optimism, adaptability, initiative, tolerance, organisational skills, being a team worker, keeping within professional boundaries, assertiveness, humour, and a sense of selfworth. Challenges in the workplace included information overload, time pressures, environmental issues in rural locations, workload, poor communication, and challenging patients. Workplace promoters included a team approach, inclusion within the wider team and a secure base, buffers (or shock absorbers), the ability to say 'no', time for reflection, and personal and social factors, such as support from friends and family, and leisure activities. Developing 
resilience was considered to be possible through experience, learning from others, and formal training.

Based on these findings, the authors propose a model of health professionals resilience in the community setting that concurs with the existing literature but adds further clarity to the concept of personal traits being synergistic with the workplace and social network. These facilitate adaptability and enable individual health professionals to cope with the adversity that is part of the everyday experience of those working in challenging environments.

\section{Strengths and limitations}

A strength of this study was the use of an inductive qualitative approach, which allowed participants to contribute their thoughts and experiences as they were generated in the focus group setting. The interest of participants was obvious through the richness of discussions. Focus groups were fruitful, with participants generating ideas from each other and endorsing or expanding on the views expressed by others. Holding focus groups in practice settings ensured that participants were more relaxed. A few participants attended late because they were held up with patientrelated tasks. This in itself generated discussion about everyday challenges.

However, a weakness of having those that work together in the same group was that they were less likely to be negative about each other or even admit if they thought they were not resilient.

A range of settings (rural and deprived) and staff ensured a range of experience was covered. However, there may be subtle differences between professional groups (GPs, nurses, and pharmacists) that were not identified in this combined approach. Pharmacists proved more difficult to recruit, potentially because they work in isolation and it is therefore more challenging for them to attend. Another limitation was the relatively small sample size and participants were from one geographical area in Scotland, albeit a large area. Healthcare systems differ in Scotland from other parts of the UK, which may have influenced the findings. However, the findings concur with the existing literature on health professional resilience, indicating that primary care is not overtly different from other areas of health care.

\section{Comparison with existing literature}

Some of the identified characteristics of resilient health professionals have been identified previously. Jensen and colleagues also noted that, to maintain a high quality of patient care, it is essential that health professionals have the ability to prioritise and plan workloads to achieve balance. ${ }^{16}$ The importance of being able to focus and deal with a problem, then move on, or 'bounce back' in the face of adversity was noted, which concurred with previous findings. ${ }^{2}$ The importance of clear professional boundaries as noted here was also noted previously as a way to mitigate against the volume and intensity of the workload. 16

There was a strong organisational component, which included systems that allow for 'buffering' when normal working practice is challenged; for example, during unexpected staff shortages. A sense of control over the working schedule was previously found to be a strong predictor of emotional resilience. ${ }^{8}$ Resilience at work can be promoted through efficient organisation structures, with forward planning and prioritisation, as well as using humour and having supportive colleagues. These were also identified in the literature. ${ }^{4,13}$ Indeed, in this study the overwhelming importance of teamwork was strongly emphasised.

Similarly, when considering threats to resilience, previous research found that one of the largest threats to resilience was the volume of paperwork and information overload..$^{10}$ In this study it was noted that this could detract from essential time for recovery and reflection, which is needed after dealing with difficult situations.

One of the promoters of resilience in this study was having, and being able to protect, a balance between work and family life. This has been noted previously. ${ }^{16}$ Leisure and social pursuits play an important role in promoting resilience as they can help divert the mental focus from work stressors. Similarly, Zwack and Schweitzer found physical activities reduced tension and cultural pursuits helped put professional stressors into perspective. ${ }^{4}$ Having a good social network was noted, which corroborates previous findings that supportive friends and family strongly predicts high levels of resilience. ${ }^{3}$

Surprisingly, the question of more challenging patients was only raised in the rural groups and not in the groups covering deprived areas. The reasons behind difficult patients in the rural groups was that late presentation of conditions required more detailed investigations and referrals, which brought with them considerable paperwork. This has also been noted in previous research on rural workload pressures. ${ }^{12}$ Nothing was raised about the behaviour of 
Figure 1. A model of health professionals' resilience in primary care.

\section{Funding}

Funded by NHS Grampian Endowment Fund (reference 12/42).

\section{Ethical approval}

Approved By University of Aberdeen College Ethical Review Board (reference CERB/2014/10/1135)

\section{Provenance}

Freely submitted; externally peer reviewed.

\section{Competing interests}

The authors have declared no competing interests.

\section{Acknowledgements}

The research team thank all participants who attended the focus group sessions. Thanks also go to Dr Tim Jones and Dr Douglas Nicol for their help and support. The team also thank NHS Grampian Endowments for funding the study.

\section{Discuss this article}

Contribute and read comments about this article: bjgp.org/letters

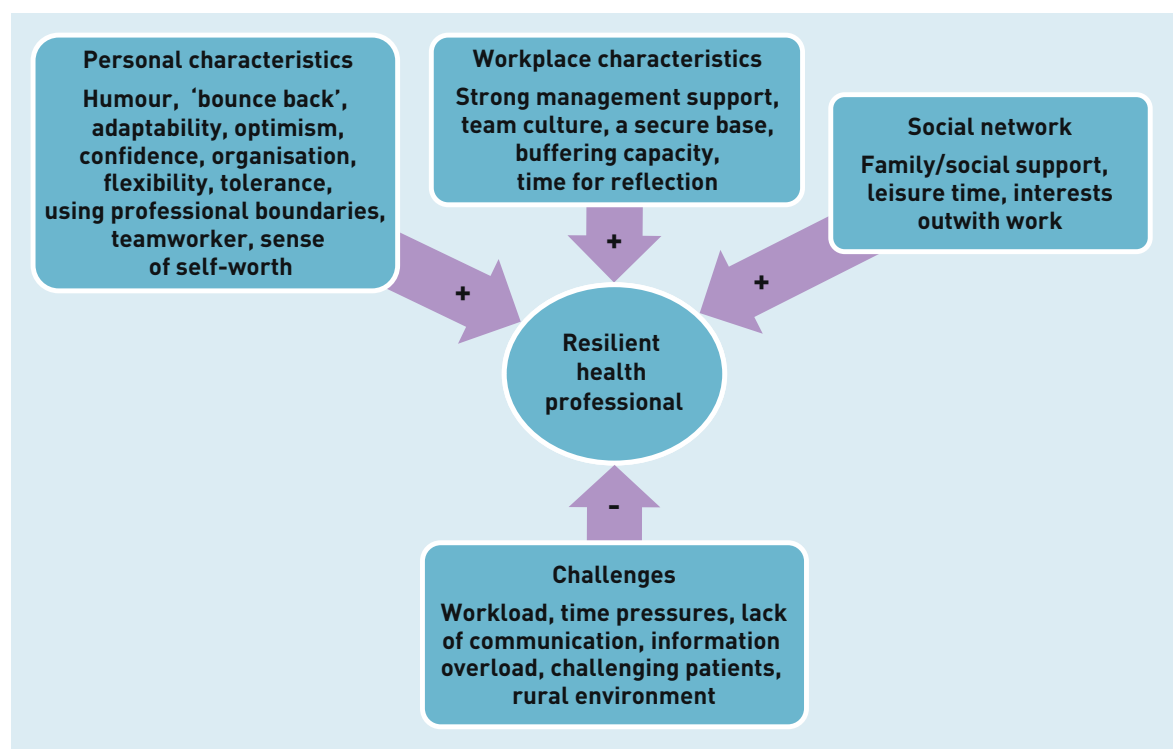

challenging patients in any of the groups.

\section{Implications for research and practice}

A model is now proposed based on this study's findings to inform future research and developments to increase resilience (Figure 1). By constructing a model it will be possible to frame future research and measurement in a way that enables the concept of professional resilience to be approached in a similar way. Identified themes can be framed in terms of personal, workplace, and social, with a strong synergistic link between these. This chimes with Lown and colleagues proposed model of resilience, with individual resilience supported by collective resilience (that is, professional structures) and broader public support. ${ }^{17}$

The authors propose, based on this qualitative evidence, that for a health professional working in a community setting to be resilient land probably also in non-community settings), their personal resilience must align with workplace resilience. It is this supportive workplace and/or a strong social network that enables the 'bouncing back' and adaptability that are so key to resilient health professionals. Challenges and enablers can work at a personal or workplace level. Importantly, a health professional's resilience is considered to be more than just a personal trait and can be developed formally through training and informally through observing and learning from others.

A further step would be to consider how current training lundergraduate and postgraduate) and support of health professionals draw on these concepts, whether explicitly or implicitly. The construction of a measure using this model as a framework would enable future quantification of professional resilience. Such a measure could be validated against existing measures of burnout. 


\section{REFERENCES}

1. Fertleman $\mathrm{C}$, Carroll W. Protecting students and promoting resilience. BMJ 2013; 347: f5266

2. Jackson D, Firtko A, Edenborough M. Personal resilience as a strategy for surviving and thriving in the face of workplace adversity: a literature review. $J$ Adv Nurs 2007; 60(1): 1-9.

3. Taku K. Relationships among perceived psychological growth, resilience and burnout in physicians. Pers Individ Dif 2014; 59: 120-123.

4. Zwack J, Schweitzer J. If every fifth physician is affected by burnout, what about the other four? Resilience strategies of experienced physicians. Acad Med 2013; 88(3): 382-389.

5. Cooke GP, Doust JA, Steele MC. A survey of resilience, burnout, and tolerance of uncertainty in Australian general practice registrars. BMC Med Educ 2013; DOI: 10.1186/1472-6920-13-2.

6. Stevenson AD, Phillips CB, Anderson KJ. Resilience among doctors who work in challenging areas: a qualitative study. Br J Gen Pract 2011; DOI: 10.3399/ bjgp11X583182.

7. Robertson HD, Elliott AM, Burton $C$, et al. Resilience in primary healthcare professionals: a systematic review. BJGP2016; DOI: 10.3399/bjgp16X685285

8. Keeton K, Fenner DE, Johnson TR, Hayward RA. Predictors of physician career satisfaction, work-life balance, and burnout. Obstet Gynecol 2007; 109(4): 949-955.

9. Skovholt TM, Trotter-Mathison M. The resilient practitioner: burnout prevention and self-care strategies for counselors, therapists, teachers, and health professionals. 2nd edn. New York, NY: Routledge, 2011.

10. Lee FJ, Stewart M, Brown JB. Stress, burnout, and strategies for reducing them: what's the situation among Canadian family physicians? Can Fam Physician 2008; 54(2): 234-235.

11. Watt G, Brown G, Budd J, et al. General practitioners at the deep end: the experience and views of general practitioners working in the most severely deprived areas of Scotland. Occas Pap R Coll Gen Pract 2012; 89: i-viii, 1-40.

12. Iversen $L$, Farmer JC, Hannaford PC. Workload pressures in rural general practice: a qualitative investigation. Scand J Prim Health Care 2002; 20(3): 139-144.

13. Bowden GE, Smith JCE, Parker PA, Boxall MJC. Working on the edge: stresses and rewards of work in a front-line mental health service. Clin Psych Psychother 2014; DOI: 10.1002/cpp.1912.

14. Scottish Government. Scottish index of multiple deprivation 2012. http://simd. scotland.gov.uk/publication-2012/ (accessed 18 Apr 2016).

15. Scottish Government. Scottish Government urban/rural classification. http:// wuw.gov.scot/Topics/Statistics/About/Methodology/UrbanRuralClassification laccessed 18 Apr 2016).

16. Jensen PM, Trollope-Kumar K, Waters H, Everson J. Building physician resilience. Can Fam Physician 2008; 54(5): 722-729.

17. Lown M, Lewith G, Simon C, Peters D. Resilience: what is it, why do we need it, and can it help us? Br J Gen Pract 2015; DOI: 10.3399/bjgp15X687133. 